Published in final edited form as:

Dev Biol. 2019 February 15; 446(2): 151-158. doi:10.1016/j.ydbio.2018.12.021.

\title{
Draxin alters laminin organization during basement membrane remodeling to control cranial neural crest EMT
}

\author{
Erica J. Hutchins ${ }^{1}$ and Marianne E. Bronner ${ }^{1,2}$ \\ ${ }^{1}$ Department of Biology and Biological Engineering, California Institute of Technology, $1200 \mathrm{E}$ \\ California Blvd, Pasadena, CA 91125, USA.
}

\begin{abstract}
Premigratory neural crest cells arise within the dorsal neural tube and subsequently undergo an epithelial-to-mesenchymal transition (EMT) to leave the neuroepithelium and initiate migration. Draxin is a Wnt modulator that has been shown to control the timing of cranial neural crest EMT. Here we show that this process is accompanied by three stages of remodeling of the basement membrane protein laminin, from regression to expansion and channel formation. Loss of Draxin results in blocking laminin remodeling at the regression stage, whereas ectopic maintenance of Draxin blocks remodeling at the expansion stage. The latter effect is rescued by addition of Snail2, previously shown to be downstream of Draxin. Our results demonstrate an essential function for the Wnt modulator Draxin in regulating basement membrane remodeling during cranial neural crest EMT.
\end{abstract}

\section{Keywords}

Neural crest; Laminin; Draxin; Snail2; Epithelial-to-Mesenchymal Transition

\section{INTRODUCTION}

Neural crest (NC) cells undergo a spatiotemporally regulated epithelial-to-mesenchymal transition (EMT) to separate from the neuroepithelium and become migratory. The process of EMT involves first breaking cell-cell and cell-basement membrane connections to detach from the neuroepithelium and delaminate (Gouignard et al., 2018; Nieto et al., 2016).

Delamination and onset of migration involves intrinsic changes in cell polarity and cell surface proteins, including cadherins and integrins, as well as remodeling of the basement membrane and interactions with interstitial extracellular matrix (ECM) components (Christian et al., 2013; Duband, 2006; Perris, 1997). In particular, laminin is a major component of both the basement membrane and interstitial ECM that plays an essential role in regulating cranial NC migration (Coles et al., 2006; Duband and Thiery, 1987; Perris and Perissinotto, 2000).

\footnotetext{
${ }^{2}$ Correspondence to Marianne E. Bronner: mbronner@caltech.edu.

Publisher's Disclaimer: This is a PDF file of an unedited manuscript that has been accepted for publication. As a service to our customers we are providing this early version of the manuscript. The manuscript will undergo copyediting, typesetting, and review of the resulting proof before it is published in its final citable form. Please note that during the production process errors may be discovered which could affect the content, and all legal disclaimers that apply to the journal pertain.
} 
During EMT, cells begin the delamination process by down-regulating laminin (Zeisberg and Neilson, 2009). Interestingly, members of the Snail family of transcription factors have been shown to directly repress transcription of laminin alpha5 (Takkunen et al., 2008), which has a critical function in cranial NC migration (Coles et al., 2006). Snail genes are well-known regulators of EMT via transcriptional repression in both cancer cells and neural crest cells (Cano et al., 2000; Strobl-Mazzulla and Bronner, 2012; Taneyhill et al., 2007). Thus, there appears to be an important link between intrinsic NC EMT factors and laminin expression. However, the mechanisms underlying the interplay between Snail, laminin, and basement membrane remodeling during neural crest EMT remain unclear.

We recently characterized the role of the Wnt signaling antagonist, Draxin, in regulating the timing of onset of cranial NC EMT (Hutchins and Bronner, 2018). Interestingly, the transcription factor Snail2, the only Snail gene family member expressed by chick cranial NC (del Barrio and Nieto, 2002), emerged as a likely target downstream of a transient pulse of Wnt signaling. Given that Snail genes are known to repress expression of laminin in other contexts (Takkunen et al., 2008) and laminin is an important component of the basement membrane surrounding the neural tube during NC EMT, we asked whether Draxin may play a role in the modulation of laminin during EMT. The results show that Draxin perturbation impedes basement membrane remodeling during cranial NC EMT via changes to laminin expression and/or organization. We further demonstrate that Draxin's effect on laminin is mediated, at least in part, by Snail2. Thus, modulation of Wnt signaling via Draxin and its downstream effector Snail2 plays a critical role in regulating basement membrane remodeling during neural crest EMT.

\section{MATERIALS AND METHODS}

\subsection{Cryosectioning and Immunohistochemistry}

Chicken embryos (Gallus gallus) were obtained commercially and incubated at $37^{\circ} \mathrm{C}$ to reach the desired Hamburger-Hamilton (Hamburger and Hamilton, 1951) stage as indicated. Embryos were fixed overnight at $4{ }^{\circ} \mathrm{C}$ in $4 \%$ paraformaldehyde in sodium phosphate buffer, then cryosectioned and immunostained as described (Hutchins and Bronner, 2018). Following gelatin removal, cryosections were incubated in primary antibodies overnight at $4^{\circ} \mathrm{C}$ and secondary antibodies for one hour at room temperature. Primary antibodies and counterstains used are listed in the Key Resources Table. Species-specific secondary antibodies were labeled with Alexa Fluor 488, 568, and 647 (Invitrogen). Prior to imaging, coverslips were mounted with Fluoromount-G (SouthernBiotech). Because Draxin expression has no effect on the early neural crest marker Pax7 (Hutchins and Bronner, 2018), we elected to use Pax7 throughout as a general neural crest label to assess effects of gene perturbation on basement membrane remodeling in the context of neural crest migration defects. Thus, defects in EMT could be attributed to changes to neural crest migration in general, and not to changes to a known/established target.

\subsection{Electroporations}

As described previously (Hutchins and Bronner, 2018; Sauka-Spengler and Barembaum, 2008; Simoes-Costa et al., 2015), dissected embryos were electroporated ex ovo at 
Hamburger-Hamilton (HH) stage HH4. Following injection of expression constructs or morpholino oligomers, embryos were electroporated using platinum electrodes (5 pulses, $6.0 \mathrm{~V}, 30 \mathrm{~ms}$ duration at $100 \mathrm{~ms}$ intervals), and cultured at $37^{\circ} \mathrm{C}$ in albumin $/ 1 \%$ penicillin/ streptomycin to indicated $\mathrm{HH}$ stages.

\subsection{Expression constructs and Morpholino Oligomers}

Draxin and Snail2 knockdown using translation-blocking antisense morpholinoelectroporation (Draxin MO and Snail2 MO, respectively, Key Resources Table; GeneTools; co-electroporated with pCIG), and Draxin overexpression using the DraxinFLAG expression construct, were performed as described (Hutchins and Bronner, 2018; Taneyhill et al., 2007). The Draxin-FLAG construct contains coding for the secreted, fulllength chick Draxin protein with a C-terminal FLAG epitope tag (Hutchins and Bronner, 2018), and a downstream internal ribosomal entry site (IRES) that drives expression of a separate Histone H2B-RFP. RFP nuclear fluorescence is used only as an indicator of successful electroporation. Control reagents were described previously (Hutchins and Bronner, 2018), and are listed in the Key Resources Table. In order to generate the NC1Snail2 construct, the coding for V5-tagged Snail2 was PCR amplified from pCIG-V5Snail2-IRES-nls-GFP (Liu et al., 2013). This V5-Snail2 fragment was then cloned into the NC1.1 M3 construct (Simoes-Costa et al., 2012), following excision of GFP by $\mathrm{NcoI}$ and $X b a I$. All constructs and primers (Integrated DNA Technologies) are listed in the Key Resources Table.

\subsection{Microscope image acquisition}

Confocal-like images were acquired with structured illumination microscopy to generate optical sections, using a Zeiss Imager.M2 with an ApoTome. 2 module and Zen 2 Blue software, with a Zeiss Plan-Apochromat 20x objective/0.8 NA, and a Zeiss EC PlanNEOFLUAR 40x oil objective/1.3 NA. Z-stacks were taken at $0.55 \mu \mathrm{m}$ (20x objective) and $0.325 \mu \mathrm{m}$ (40x objective) intervals and displayed as maximum intensity projections. Images were minimally processed for brightness and contrast using Adobe Photoshop CC.

\subsection{Statistical analysis}

Statistical analyses were performed using GraphPad Prism 7. $P$ values were calculated using an unpaired, two-tailed t-test and are indicated in the text. Data are presented as the percentage of the total number of sections displaying the specified phenotype ( $\mathrm{n} \geq 8$ sections among 5 embryos, per condition). An "incomplete channel" was scored if laminin appeared to partially block channel opening, but Pax7+ neural crest cells were able to pass laterally beyond the laminin blockage. "No channel" was scored if laminin formed a complete barrier beyond which Pax $7+$ cells could not migrate. Multiple sections from multiple embryos were factored into our analysis to account for section-to-section differences and biological variability. Further, this variation is why we chose not to quantify fluorescence intensity in the neural crest region, but rather characterize gross morphological changes to the organization of the basement membrane (i.e. channel or no channel) in order to simplify phenotypic interpretation and reduce the impact of technical and biological variability. 


\section{RESULTS}

\subsection{Cranial neural crest migrates through a laminin channel during EMT}

In the avian cranial $\mathrm{NC}$, the events of EMT and neural tube closure progress simultaneously. As the neural tube undergoes separation from the non-neural ectoderm, premigratory NC cells in this region delaminate and complete EMT to migrate dorsolaterally between the newly-formed overlying dorsal ectoderm and the underlying ventral mesoderm (Baker et al., 1997; Duband, 2006; Theveneau and Mayor, 2012). The separation of the neural tube from the non-neural ectoderm occurs via a remodeling of the laminin-rich basement membrane from a single, continuous structure into distinct dorsal ectodermal and neural tube basement membranes (Duband and Thiery, 1987).

In order to correlate events of cranial NC EMT with reorganization of the basement membrane, we examined the expression of laminin (as a marker for the basement membrane) and the neural crest marker Pax7 across several developmental time points (Fig. 1, Fig. S1). Immunohistochemical analysis of transverse sections through wild-type chick mesencephalon and rostral hindbrain revealed dynamic rearrangement of the basement membrane correlating with discrete stages of cranial neural crest EMT. Between HamburgerHamilton stages 8+ and 9- (HH8+/9-), as specified premigratory NC cells were localized to the dorsal aspect of the neural tube, the basement membrane was remodeled to form a space between the non-neural ectoderm and neural tube, which were tightly apposed at HH8+. This remodeling served to increase the angle between the non-neural ectoderm and neuroepithelium basement membranes (Fig. 1A-B, arrowheads). At HH9, as cranial NC began delaminating from the neuroepithelium, the basement membrane at the junction of the neural tube and ectoderm remained continuous, despite expanding to encapsulate newly delaminated NC, apparently creating a barrier that blocked NC entry into the dorsolateral migratory route (Fig. 1C). By HH9+, the lateral laminin barrier disappeared to generate separate ectodermal and neural tube basement membranes. Concomitantly, cranial NC cells completed EMT and mesenchymalized to migrate away from the neural tube (Hutchins and Bronner, 2018). Because the basement membranes of the ectoderm and neural tube remained in close proximity, this separation formed a laminin-lined "channel", through which the cranial NC migrated (Fig. 1D). Thus, during cranial NC EMT, basement membrane remodeling occurs in three successive stages, which we term "Regression" (Fig. 1B"), "Expansion" (Fig. 1C"), and "Channel Formation" (Fig. 1D").

\subsection{Draxin knockdown impedes basement membrane remodeling during EMT}

We previously identified a critical role of the Wnt antagonist, Draxin, in the regulation of cranial NC EMT; loss of Draxin induced premature NC exit from the neuroepithelium, though the cells failed to complete EMT and become migratory (Hutchins and Bronner, 2018). To determine whether Draxin played a role in basement membrane remodeling, we examined transverse sections of embryos unilaterally electroporated with either control morpholino (MO) (Fig. 2A-B) or a translation-blocking Draxin MO (Fig. 2C-D). Whereas control MO electroporation yielded no significant change in the incidence of laminin channel formation compared to the contralateral uninjected side ( $p=0.63$; Fig. 2E), loss of Draxin significantly inhibited laminin channel formation $(p<0.0001$; Fig. 2F). Examination 
of the Draxin knockdown phenotype at stage HH9+ showed a continuous basement membrane (Fig. 2D, arrowhead), with laminin expression resembling a stage HH9- wild type phenotype (Fig. 1B), with neural tube closure appearing to progress normally; interestingly, this is also the stage of onset of Draxin expression (Hutchins and Bronner, 2018). We further examined earlier stages of development (HH9-/9), and observed defects in laminin organization as early as HH9 (Fig. S2). These data suggest that Draxin knockdown halts basement membrane remodeling at the "Regression" stage. Thus, Draxin is necessary for complete basement membrane reorganization during cranial neural crest EMT.

\subsection{Ectopic maintenance of Draxin blocks laminin channel formation during EMT}

Draxin is expressed in a transient head-to-tail wave within the premigratory NC, and its downregulation mirrors initiation of cranial NC emigration. Furthermore, ectopic overexpression of Draxin after its endogenous downregulation results in inhibited cranial NC delamination and migration (Hutchins and Bronner, 2018). Thus, we sought to assess the effects of ectopic Draxin overexpression on laminin expression during EMT. Following unilateral electroporation of pCIH2B-RFP as a control (Fig. 3A-B) or a Draxin-FLAG overexpression construct (Hutchins and Bronner, 2018; Fig. 3C-D), we examined transverse sections of embryos for effects on laminin channel formation. Control pCI-H2B-RFP electroporations had no significant effect on laminin channel formation ( $P=0.29$; Fig. 3E). In contrast, ectopic Draxin overexpression significantly reduced the incidence of complete channel formation $(P<0.0001$; Fig. 3F); examination of the Draxin-FLAG phenotype at stage $\mathrm{HH} 9+$ revealed a physical blockage of the laminin channel due to aberrant laminin organization (Fig. 3D, arrowhead), resembling a stage HH9 wild type phenotype (Fig. 1C). These data suggest that ectopic maintenance of Draxin halts basement membrane remodeling at the "Expansion" stage, inhibiting the dissolution of the lateral laminin barrier, which creates a physical block to the migration of cranial NC away from the neural tube.

\subsection{Perturbation of Snail2 alters laminin channel formation during EMT}

We have previously demonstrated that Draxin overexpression inhibited Snail2 in cranial NC (Hutchins and Bronner, 2018). Given that Snail family members have been implicated in both EMT and the transcriptional control of laminin (Cano et al., 2000; Takkunen et al., 2008; Zeisberg and Neilson, 2009), we asked whether the effects of Draxin on laminin channel formation and basement membrane remodeling in cranial $\mathrm{NC}$ are mediated by Snail2. To this end, we performed unilateral electroporation of a translation-blocking Snail2 MO (Taneyhill et al., 2007) and observed a significant reduction in the number of Snail2+ cells on the MO-electroporated side (Fig. S3A-C; *, $P=0.004$, two tailed $t$-test). We next examined transverse sections of Snail2 MO-electroporated embryos for effects on laminin channel formation, and found that Snail2 knockdown significantly impeded laminin channel formation (Fig. 4A-C, Fig. S3D-G; *, $P=0.0007$, two tailed $t$-test). To further confirm that Draxin's effect on basement membrane remodeling is mediated at least in part by Snail2, we generated a Snail2 overexpression construct (NC1-Snail2) in which the FoxD3 NC1 enhancer (Simoes-Costa et al., 2012) drives expression of V5-tagged, full-length chick Snail2 (Fig. S4). This construct restricts expression of Snail2 to the cranial NC following specification, in order to bypass potential deleterious effects on earlier induction and specification events. When co-electroporated with Draxin-FLAG (Fig. D-E), we observed 
complete rescue of laminin channel formation (Fig. 4F; compare to Fig. 3). Together, these data suggest that Draxin controls the progression of basement membrane remodeling, in large part, via modulation of Snail2.

\section{DISCUSSION}

The canonical Wnt antagonist, Draxin, has critical functions in regulating the progression of cranial NC EMT. A transient pulse of Draxin expression tightly coordinates the events of cranial NC delamination and mesenchymalization, to allow successful migration away from the neural tube (Hutchins and Bronner, 2018). Here, we demonstrate a novel function of Draxin during EMT - in regulation of basement membrane remodeling. Our data provide a mechanistic link between Wnt signaling modulation and progressive laminin reorganization to restructure the basement membrane.

Combined with our prior study (2018), our results suggest the following model for the interplay between Snail2, laminin, and basement membrane remodeling during cranial NC EMT. Under wild-type conditions, endogenous Draxin expression within the premigratory cranial neural crest attenuates Wnt signaling, and by extension, the Wnt target gene Snail2; reduced Wnt signaling maintains the basement membrane in a single, continuous layer that is able to progress through the "Regression" and "Expansion" stages. As Draxin is endogenously downregulated, Wnt signaling increases, allowing intrinsic NC EMT factors (e.g. Snail2, etc.) to dissolve the lateral laminin barrier (presumably via both transcriptional and post-translational regulation) and complete channel formation.

With loss of Draxin, Wnt signaling increases prematurely and halts laminin reorganization. We hypothesize this abrogation of basement membrane remodeling may be in part due to reduced Cadherin6B (Cad6B) expression. Cad6B is a target of proteolytic cleavage, and it was recently shown that its cleaved fragments promote laminin degradation and basement membrane remodeling during EMT (Schiffmacher et al., 2018; Schiffmacher et al., 2014). Thus, it is possible the premature reduction of Cad6B found with loss of Draxin (Hutchins and Bronner, 2018) subsequently reduces the availability of Cad6B cleavage fragments to modulate laminin. Furthermore, because Snaill directly represses transcription of a subtype of laminin during EMT (Takkunen et al., 2008), loss of Draxin may affect laminin expression in multiple ways to impede basement membrane remodeling. With ectopic maintenance of Draxin, Wnt signaling fails to increase following NC delamination, and as such, Snail2 expression remains suppressed as Cad6B is inappropriately maintained (Hutchins and Bronner, 2018). This results in a failure to remove the laminin blockage to complete channel formation, despite an increase in Cad6B. We hypothesize that the inability to remove the laminin blockage may be due the loss of Snail2, and the resulting inability to transcriptionally repress laminin and/or degrade the physical barrier to migration. This argument is strengthened by the ability of Snail2 overexpression to rescue laminin channel formation from Draxin overexpression. However, there may also be a reduction in the cleavage of Cad6B, subsequently impeding laminin degradation; examination of whether Draxin perturbation also affects expression of A Disintegrin and Metalloproteinases (ADAMs) or matrix metalloproteinases (MMPs), which themselves target Cad6B (Schiffmacher et al., 2014), would help resolve this question. In addition, laminin channel 
formation may require not only modification to the ECM components of the basal lamina via proteolytic cleavage, but also the force of migratory neural crest cells pushing against the laminin barrier to facilitate remodeling (Clay and Halloran, 2010, 2011). Parsing whether Draxin perturbation alters proteolytic cleavage of Cad6B, tensile strength of the basement membrane, or motile force of the neural crest cells themselves may provide further mechanistic insights into the effects on basement membrane remodeling during EMT.

We believe the interplay between basement membrane remodeling and neural crest migration we describe here fits nicely with recent work identifying the importance of neural crest/ECM interactions in other systems. For example, cephalic neural crest cells in Xenopus have been shown to directly interact with the ECM, are responsive to matrix/environmental stiffening, and that these mechanical cues are required for successful neural crest EMT and subsequent contact inhibition of locomotion (Barriga et al., 2018; Scarpa et al., 2015). Furthermore, it is interesting that the presence of the ECM proteoglycan versican acts as a nonpermissive substrate to confine neural crest, providing a mechanism of stream formation and directional migration (Szabo et al., 2016). This model of in vivo confinement correlates with our description of laminin channel formation, and suggests an important, conserved role for the ECM and basement membrane in both facilitating and directing neural crest EMT and migration.

In summary, our results suggest an essential function for Draxin in regulating basement membrane remodeling during cranial NC EMT. The progressive reorganization of laminin within the basement membrane correlates with the endogenous transient pulse of Draxin expression. Draxin's role as a canonical Wnt antagonist thus suggests that the events of cranial NC EMT and basement membrane remodeling are inextricably linked, and that they are tightly controlled and coordinated with canonical Wnt signaling.

\section{Supplementary Material}

Refer to Web version on PubMed Central for supplementary material.

\section{ACKNOWLEDGMENTS}

We thank Dr. M. Simoes-Costa for providing the NC1.1 M3:EGFP construct, and Dr. M. Cheung for providing the pCIG-V5-Snail2-IRES-nls-GFP construct (Addgene plasmid \# 44282). This work was supported by a National Institutes of Health grant (R01DE024157 and PO1HD037105 to M.E. Bronner), and a Ruth L. Kirschstein National Research Service Award (F32DE026355 to E.J. Hutchins). The authors declare no competing interests.

\section{REFERENCES}

Baker CV, Bronner-Fraser M, Le Douarin NM, Teillet MA, 1997 Early- and late-migrating cranial neural crest cell populations have equivalent developmental potential in vivo. Development 124, 3077-3087. [PubMed: 9272949]

Barriga EH, Franze K, Charras G, Mayor R, 2018 Tissue stiffening coordinates morphogenesis by triggering collective cell migration in vivo. Nature 554, 523-527. [PubMed: 29443958]

Cano A, Perez-Moreno MA, Rodrigo I, Locascio A, Blanco MJ, del Barrio MG, Portillo F, Nieto MA, 2000 The transcription factor snail controls epithelial-mesenchymal transitions by repressing Ecadherin expression. Nature cell biology 2, 76-83. [PubMed: 10655586] 
Christian L, Bahudhanapati H, Wei S, 2013 Extracellular metalloproteinases in neural crest development and craniofacial morphogenesis. Crit Rev Biochem Mol Biol 48, 544-560. [PubMed: 24066766]

Clay MR, Halloran MC, 2010 Control of neural crest cell behavior and migration: Insights from live imaging. Cell Adh Migr 4, 586-594. [PubMed: 20671421]

Clay MR, Halloran MC, 2011 Regulation of cell adhesions and motility during initiation of neural crest migration. Curr Opin Neurobiol 21, 17-22. [PubMed: 20970990]

Coles EG, Gammill LS, Miner JH, Bronner-Fraser M, 2006 Abnormalities in neural crest cell migration in laminin alpha5 mutant mice. Developmental biology 289, 218-228. [PubMed: 16316641]

del Barrio MG, Nieto MA, 2002 Overexpression of Snail family members highlights their ability to promote chick neural crest formation. Development 129, 1583-1593. [PubMed: 11923196]

Duband JL, 2006 Neural crest delamination and migration: integrating regulations of cell interactions, locomotion, survival and fate. Adv Exp Med Biol 589, 45-77. [PubMed: 17076275]

Duband JL, Thiery JP, 1987 Distribution of laminin and collagens during avian neural crest development. Development 101, 461-478. [PubMed: 3332259]

Gouignard N, Andrieu C, Theveneau E, 2018 Neural crest delamination and migration: Looking forward to the next 150 years. Genesis 56, e23107. [PubMed: 29675839]

Hamburger V, Hamilton HL, 1951 A series of normal stages in the development of the chick embryo. J Morphol 88, 49-92. [PubMed: 24539719]

Hutchins EJ, Bronner ME, 2018 Draxin acts as a molecular rheostat of canonical Wnt signaling to control cranial neural crest EMT. The Journal of cell biology 217, 3683-3697. [PubMed: 30026247]

Liu JA, Wu MH, Yan CH, Chau BK, So H, Ng A, Chan A, Cheah KS, Briscoe J, Cheung M, 2013 Phosphorylation of Sox9 is required for neural crest delamination and is regulated downstream of BMP and canonical Wnt signaling. Proceedings of the National Academy of Sciences of the United States of America 110, 2882-2887. [PubMed: 23382206]

Nieto MA, Huang RY, Jackson RA, Thiery JP, 2016 Emt: 2016. Cell 166, 21-45. [PubMed: 27368099]

Perris R, 1997 The extracellular matrix in neural crest-cell migration. Trends Neurosci 20, 23-31. [PubMed: 9004416]

Perris R, Perissinotto D, 2000 Role of the extracellular matrix during neural crest cell migration. Mechanisms of development 95, 3-21. [PubMed: 10906446]

Sauka-Spengler T, Barembaum M, 2008 Gain- and loss-of-function approaches in the chick embryo. Methods in cell biology 87, 237-256. [PubMed: 18485300]

Scarpa E, Szabo A, Bibonne A, Theveneau E, Parsons M, Mayor R, 2015 Cadherin Switch during EMT in Neural Crest Cells Leads to Contact Inhibition of Locomotion via Repolarization of Forces. Developmental cell 34, 421-434. [PubMed: 26235046]

Schiffmacher AT, Adomako-Ankomah A, Xie V, Taneyhill LA, 2018 Cadherin-6B proteolytic Nterminal fragments promote chick cranial neural crest cell delamination by regulating extracellular matrix degradation. Developmental biology.

Schiffmacher AT, Padmanabhan R, Jhingory S, Taneyhill LA, 2014 Cadherin-6B is proteolytically processed during epithelial-to-mesenchymal transitions of the cranial neural crest. Mol Biol Cell 25, 41-54. [PubMed: 24196837]

Simoes-Costa M, Stone M, Bronner ME, 2015 Axud1 Integrates Wnt Signaling and Transcriptional Inputs to Drive Neural Crest Formation. Developmental cell 34, 544-554. [PubMed: 26256212]

Simoes-Costa MS, McKeown SJ, Tan-Cabugao J, Sauka-Spengler T, Bronner ME, 2012 Dynamic and differential regulation of stem cell factor FoxD3 in the neural crest is Encrypted in the genome. PLoS genetics 8, e1003142. [PubMed: 23284303]

Strobl-Mazzulla PH, Bronner ME, 2012 A PHD12-Snail2 repressive complex epigenetically mediates neural crest epithelial-to-mesenchymal transition. The Journal of cell biology 198, 999-1010. [PubMed: 22986495]

Szabo A, Melchionda M, Nastasi G, Woods ML, Campo S, Perris R, Mayor R, 2016 In vivo confinement promotes collective migration of neural crest cells. The Journal of cell biology 213, 543-555. [PubMed: 27241911] 
Takkunen M, Ainola M, Vainionpaa N, Grenman R, Patarroyo M, Garcia de Herreros A, Konttinen YT, Virtanen I, 2008 Epithelial-mesenchymal transition downregulates laminin alpha5 chain and upregulates laminin alpha4 chain in oral squamous carcinoma cells. Histochem Cell Biol 130, 509-525. [PubMed: 18496706]

Taneyhill LA, Coles EG, Bronner-Fraser M, 2007 Snail2 directly represses cadherin6B during epithelial-to-mesenchymal transitions of the neural crest. Development 134, 1481-1490. [PubMed: 17344227]

Theveneau E, Mayor R, 2012 Neural crest delamination and migration: from epitheliumtomesenchyme transition to collective cell migration. Developmental biology 366, 34-54. [PubMed: 22261150]

Zeisberg M, Neilson EG, 2009 Biomarkers for epithelial-mesenchymal transitions. J Clin Invest 119, 1429-1437. [PubMed: 19487819] 


\section{HIGHLIGHTS}

- Cranial neural crest migrate through a laminin-rich basement membrane channel

- Perturbation of Draxin, a Wnt antagonist, alters laminin channel formation

- Draxin's effect on laminin channel formation is largely mediated by Snail2 

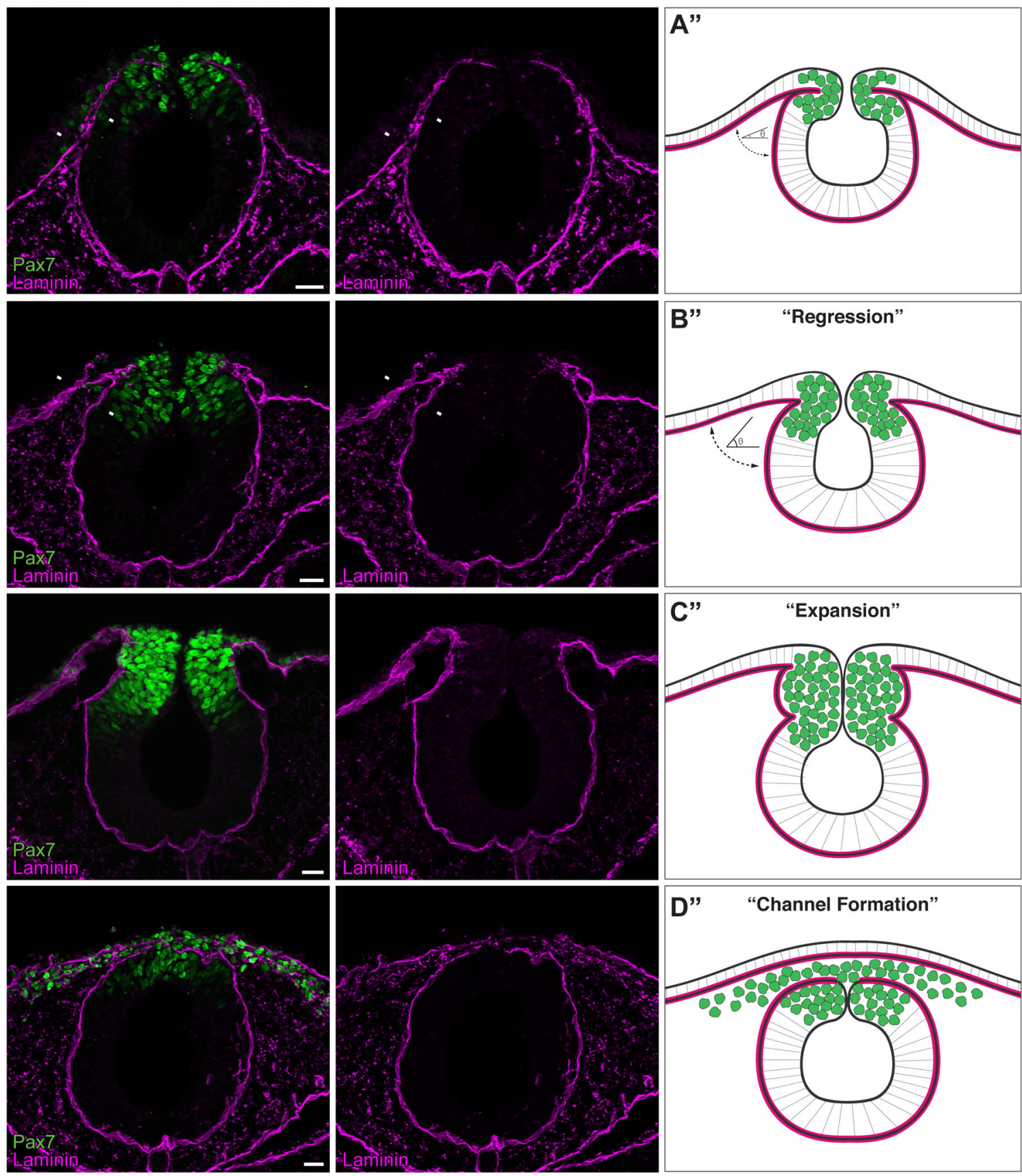

Figure 1. The basement membrane is remodeled during cranial neural crest EMT.

(A-D) Immunostaining for Pax7 (green) and laminin (magenta) in cross sections of HH8+ (A), HH9- (B), HH9 (C), and HH9+ (D) embryos. Arrowheads in (A') and (B') indicate apposed and non-apposed basement membranes, respectively. Scale bar, $20 \mu \mathrm{m}$. Schematics (A"-D") summarize immunostaining data ( $\mathrm{n}=12$ sections, 3 embryos per stage), where green circles represent neural crest cells and magenta lines indicate the laminin-rich basement membrane. As development progresses from HH8+ to HH9-, a "regression" occurs, where the angle $(\theta)$ between the non-neural ectoderm basement membrane and the neuroepithelium 
basement membrane increases (A"-B"). As Pax7+ neural crest cells undergo EMT, a laminin channel forms by $\mathrm{HH} 9+$, creating a passage through which the cells are able to migrate. 
Control MO
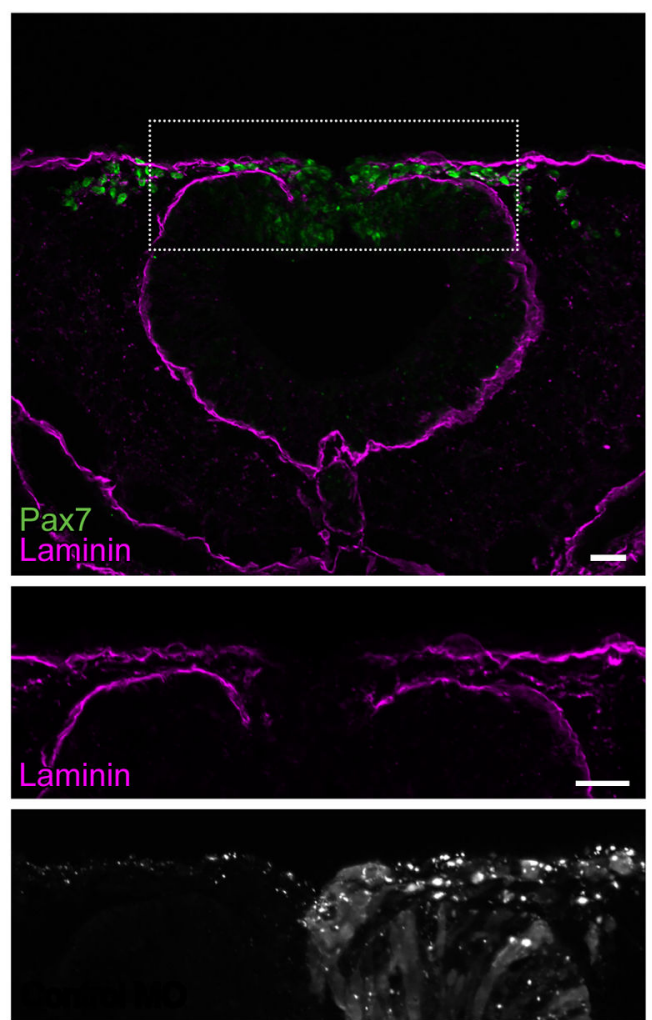

E Effect on Laminin Channel Formation

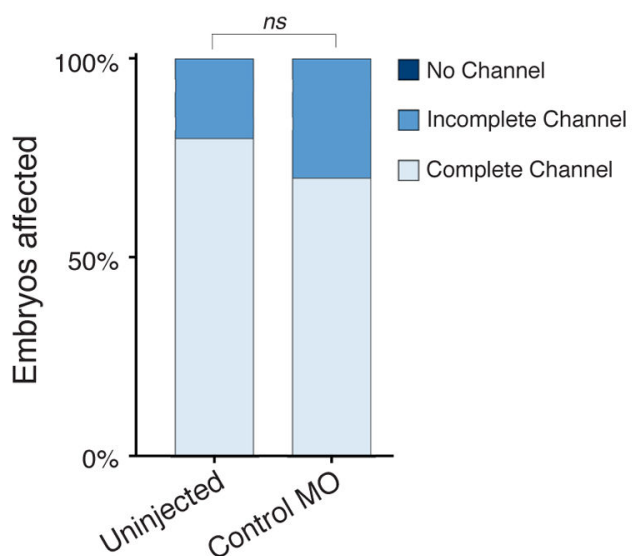
0.0001 , two-tailed $t$-test. ns, nonsignificant.

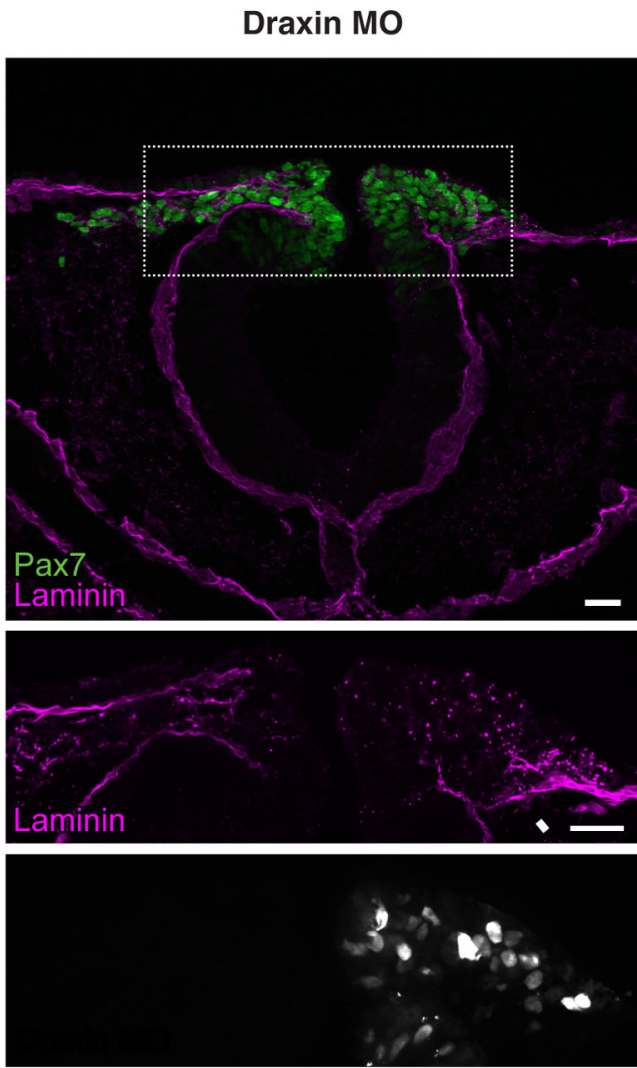

\section{F Effect on Laminin Channe Formation}

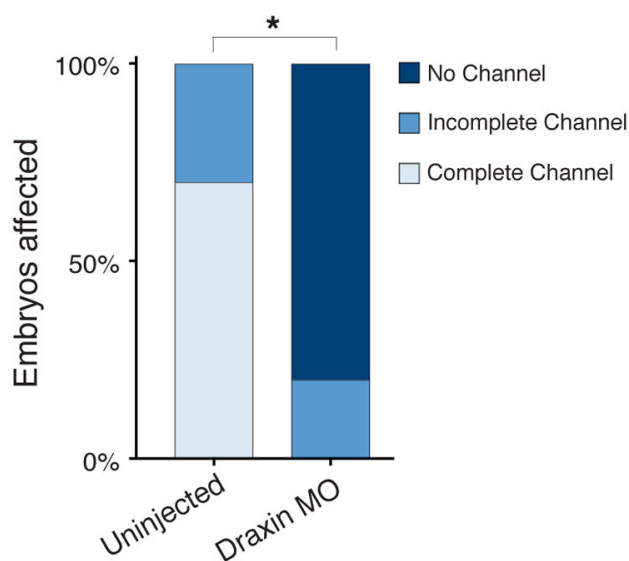

Figure 2. Draxin knockdown inhibits basement membrane remodeling during EMT. (A-D) Representative images for Pax7 (green) and laminin (magenta) immunostaining in embryos unilaterally electroporated with FITC-labeled control (A-B) or translation-blocking Draxin (C-D) morpholino (MO). Scale bar, $20 \mu \mathrm{m}$. (E-F) Quantification of the effect of MO electroporation indicated Draxin knockdown significantly reduced laminin channel formation, whereas control MO did not $(p=0.63)$. Boxes in (A,D) indicate enlarged areas in $(\mathrm{B}, \mathrm{E})$, as indicated. White arrowhead highlights area of altered laminin distribution. ${ }^{*}, p<$ 
pCl-H2B-RFP
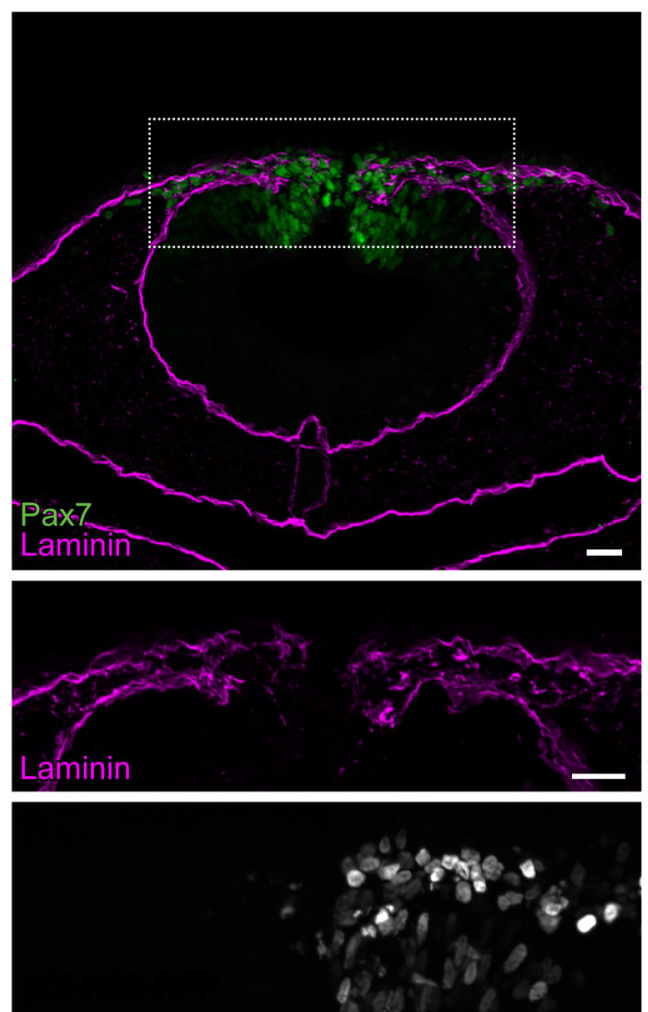

E Effect on Laminin Channel Formation

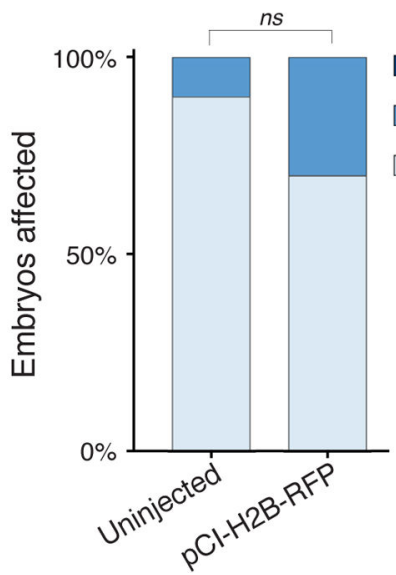

Draxin-FLAG
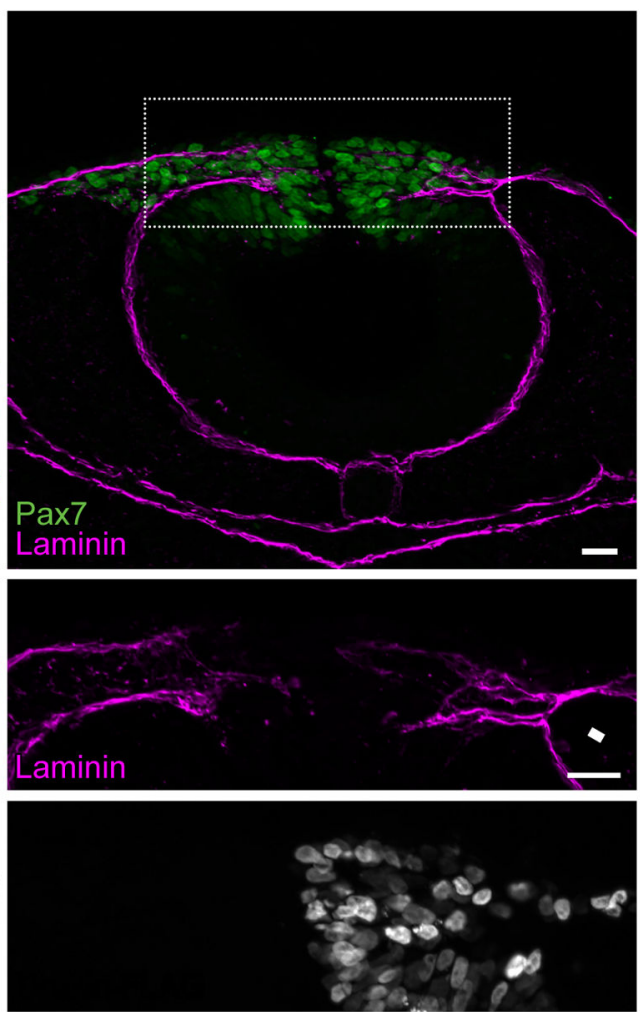

F Effect on Laminin Channel Formation

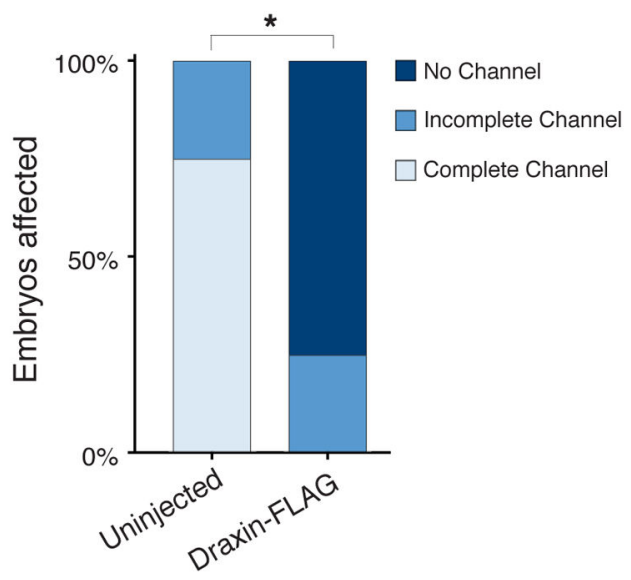

Figure 3. Ectopic maintenance of Draxin impedes laminin channel formation.

(A-D) Representative images for Pax7 (green) and laminin (magenta) immunostaining in embryos unilaterally electroporated with pCI-H2B-RFP control (A-B) or a Draxin-FLAG overexpression construct (C-D). Scale bar, $20 \mu \mathrm{m}$. (E-F) Quantification of laminin channel formation indicated that, whereas pCI-H2B-RFP electroporation had no effect on channel formation ( $p=0.29)$, Draxin-FLAG overexpression significantly inhibited channel completion due to altered laminin distribution. Boxes in $(\mathrm{A}, \mathrm{D})$ indicate zoomed areas in $(\mathrm{B}, \mathrm{E})$, as indicated. White arrowhead highlights area of altered laminin distribution. *, $p<$ 0.0001 , two-tailed $t$-test. ns, nonsignificant. 
Snail2 MO
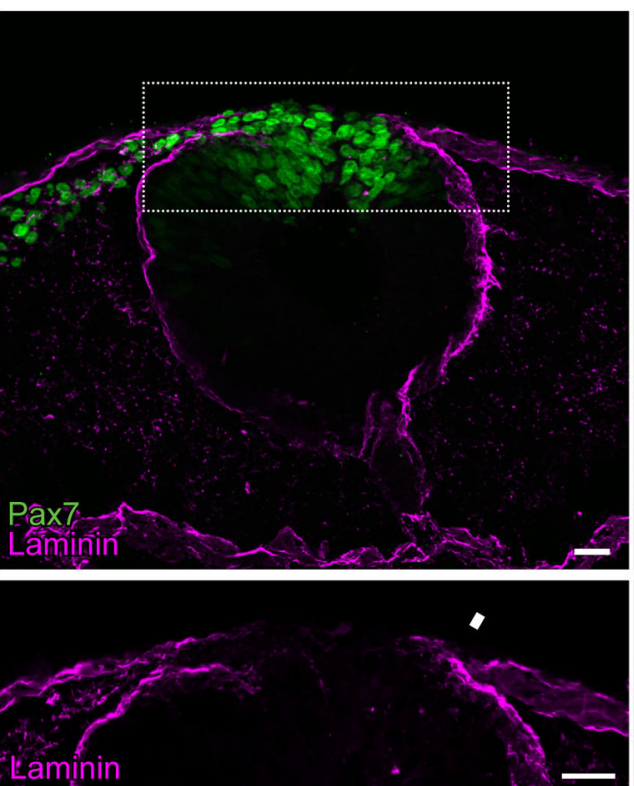

Laminin

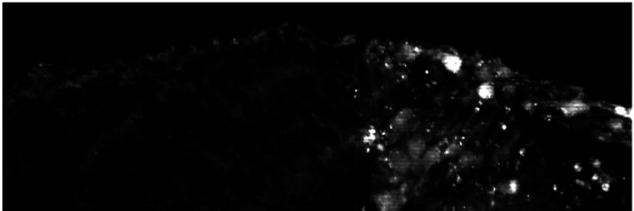

C Effect on Laminin Channel Formation

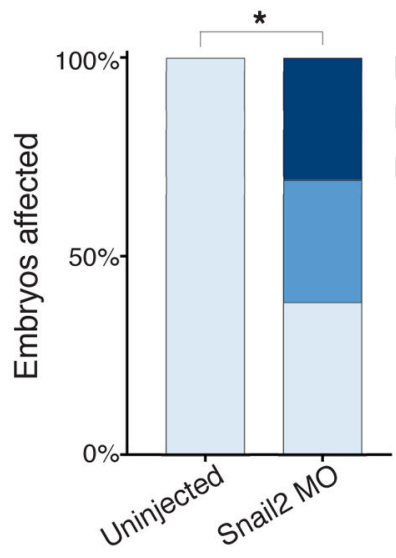

Draxin-FLAG + NC1-Snail2
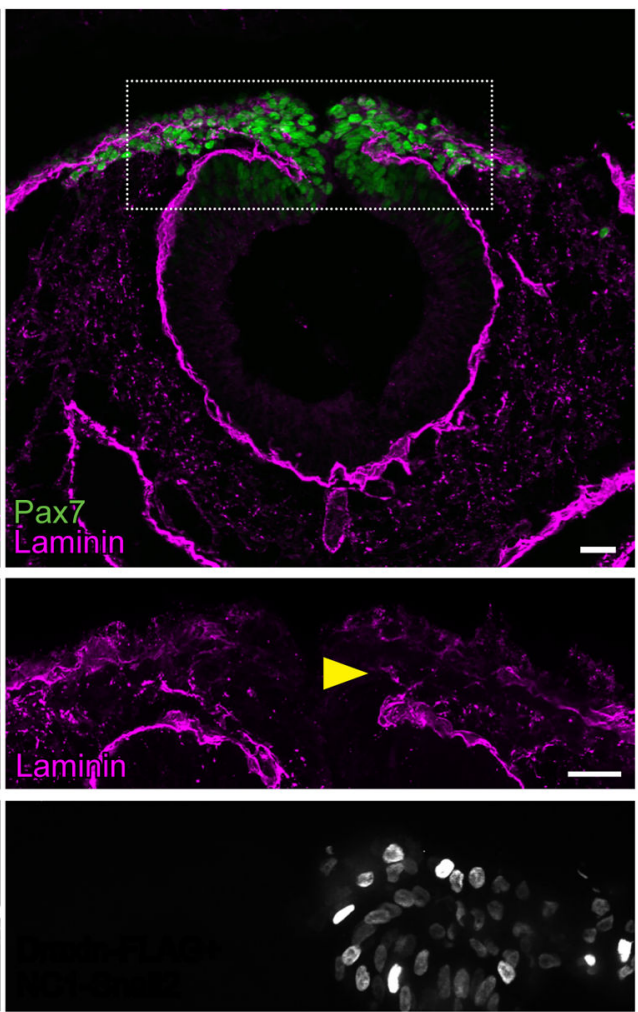

$\mathbf{F}$

Effect on Laminin Channel Formation

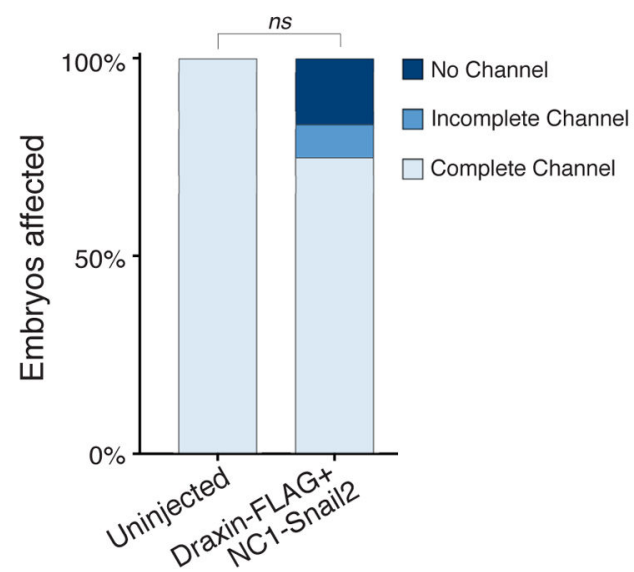

Figure 4. Modulating Snail2 alters laminin channel formation.

(A-B) Representative images for Pax7 (green) and laminin (magenta) immunostaining in embryos unilaterally electroporated with FITC-labeled translation-blocking Snail2 morpholino (MO). Scale bar, $20 \mu \mathrm{m}$. (C) Quantification of the effect of MO electroporation indicated Snail2 knockdown significantly reduced laminin channel formation. Box in (A) indicates enlarged area in (B), as indicated. White arrowhead highlights area of altered laminin distribution. *, $p<0.001$, two-tailed $t$-test. ns, nonsignificant. (D-E) Representative images for Pax7 (green) and laminin (magenta) immunostaining in embryos unilaterally coelectroporated with NC1-Snail2 and Draxin-FLAG. Nuclear fluorescence in E' indicates 
electroporated side. Scale bar, $20 \mu \mathrm{m}$. Box in (D) indicates enlarged area in (E), as indicated. Yellow arrowhead highlights rescue of channel formation. (F) Quantification of laminin channel formation indicated that NC1-Snail2 rescued the effect of Draxin-FLAG overexpression on channel formation. ns, nonsignificant $(p=0.082)$. 
KEY RESOURCES TABLE

\begin{tabular}{|c|c|c|}
\hline Reagent or resource & Source & Identifier \\
\hline \multicolumn{3}{|l|}{ Antibodies } \\
\hline Pax7; Species: Mouse IgG1 & $\begin{array}{l}\text { Developmental } \\
\text { Studies Hybridoma } \\
\text { Bank }\end{array}$ & Cat\# pax7, RRID:AB_528428 \\
\hline Pan-Laminin; Species: Rabbit & Sigma-Aldrich & Cat\# L9393 \\
\hline Fluorescein; Species: Goat & Novus Biologicals & Cat\# NB600-493 \\
\hline FLAG M2; Species Mouse IgG1 & Sigma-Aldrich & Cat\# F1804 \\
\hline V5; Species: Mouse IgG2a & Thermo & Cat\# R960-25 \\
\hline Slug (C19G7); Species: Rabbit & Cell Signaling & Cat\# 9585 \\
\hline \multicolumn{3}{|l|}{ Chemicals, Peptides, and Recombinant Proteins } \\
\hline Fluoromount-G & SouthernBiotech & Cat\# 0100-01 \\
\hline Wheat Germ Agglutinin, Alexa Fluor ${ }^{\mathrm{TM}} 488$ Conjugate & Thermo & W11261 \\
\hline DAPI (4',6-Diamidino-2-Phenylindole, Dihydrochloride) & Thermo & D1306 \\
\hline \multicolumn{3}{|l|}{ Experimental Models: Organisms/Strains } \\
\hline Gallus gallus & $\begin{array}{l}\text { Sun State Ranch } \\
\text { (Monrovia, CA, } \\
\text { USA) }\end{array}$ & N/A \\
\hline \multicolumn{3}{|l|}{ Oligonucleotides } \\
\hline Draxin morpholino w/ 3'Fluorescein: AAGGTGGAAGAAGCTGCCATAATCC & $\begin{array}{l}\text { Hutchins and } \\
\text { Bronner, 2018; } \\
\text { GeneTools }\end{array}$ & N/A \\
\hline $\begin{array}{l}\text { Standard Control morpholino w/ 3'Fluorescein: } \\
\text { CCTCTTACCTCAGTTACAATTTATA }\end{array}$ & GeneTools & N/A \\
\hline Snail2 morpholino w/ 3'Fluorescein: TCTTGACCAGGAAGGAGC & $\begin{array}{l}\text { Taneyhill et al., } \\
\text { 2007; GeneTools }\end{array}$ & N/A \\
\hline Nhel-V5-Snail2_F Primer: ATAGCTAGCGCCACCATGGGTAAGCCTATCCCTAAC & This paper; IDT & N/A \\
\hline Xbal-V5-Snail2_R Primer: ACCTCTAGATCAGTGTGCTACGCAGCAG & This paper; IDT & N/A \\
\hline \multicolumn{3}{|l|}{ Recombinant DNA } \\
\hline pCI-H2B-RFP & $\begin{array}{l}\text { Betancur et al., } \\
2010\end{array}$ & N/A \\
\hline Draxin-FLAG & $\begin{array}{l}\text { Hutchins and } \\
\text { Bronner, } 2018\end{array}$ & N/A \\
\hline NC1.1 M3:eGFP & $\begin{array}{l}\text { Simoes-Costa et } \\
\text { al., } 2012\end{array}$ & N/A \\
\hline pCIG-V5-Snail2-IRES-nls-GFP & Liu et al., 2013 & Addgene Plasmid \#44282 \\
\hline pCIG & $\begin{array}{l}\text { Megason and } \\
\text { McMahon, } 2002\end{array}$ & N/A \\
\hline NC1.1M3-Snail2 & This paper & N/A \\
\hline \multicolumn{3}{|l|}{ Software and Algorithms } \\
\hline ImageJ64 & $\mathrm{NIH}$ & N/A \\
\hline Fiji & $\begin{array}{l}\text { Schindelin et al., } \\
2012\end{array}$ & N/A \\
\hline Zen 2 Blue & Zeiss & N/A \\
\hline
\end{tabular}




\begin{tabular}{|l|l|l|}
\hline Reagent or resource & Source & Identifier \\
\hline Photoshop CC & Adobe & N/A \\
\hline
\end{tabular}

